\title{
Exclusion criteria for assuring safety of single-incision laparoscopic cholecystectomy
}

\author{
Yoshikuni Kawaguchi, Takeaki Ishizawa, Rihito Nagata, Junichi Kaneko, Yoshihiro Sakamoto, \\ Taku Aoki, Yasuhiko Sugawara, Kiyoshi Hasegawa, Norihiro Kokudo* \\ Hepato-Biliary-Pancreatic Surgery Division, Department of Surgery, Graduate School of Medicine, The University of Tokyo, \\ Tokyo, Japan.
}

\begin{abstract}
Summary Despite increasing popularity of single-incision laparoscopic cholecystectomy (SILC), indication criteria assuring safety of SILC has yet to be established. In the present study, the subjects consisted of 146 consecutive patients undergoing conventional laparoscopic cholecystectomy (CLC) or SILC. SILC was indicated after excluding patients who met following criteria: age $>75$ years, obesity, operative scar, cardiopulmonary diseases, acute cholecystitis, choledocholithiasis and abnormal bile duct anatomy. Thirty-four patients were excluded from the SILC candidates (moderate/high-risk CLC group). Among the 112 potential candidates, SILC was indicated for 23 patients $(21 \%$, SILC group) and the remaining 89 patients (79\%) underwent CLC (low-risk CLC group). In the SILC group, operation time was longer than in the low-risk CLC group (171 [113-286] vs. 126 [72-240] min, $p<0.01$ ), but the periods requiring painkiller was shorter. That led to reduced length of hospital stay compared to low-risk CLC group (2 [2-4] vs. 4 [2-12] days, $p<0.01)$. Between the low-risk CLC and moderate/high-risk CLC group, operation time was significantly longer and amount of blood loss was larger in the latter group. No complications were encountered in the SILC group. SILC can be indicated safely as far as appropriate criteria is adopted for excluding patients in whom complicated laparoscopic procedures are needed.
\end{abstract}

Keywords: Conventional multiport laparoscopic cholecystectomy, fluorescence cholangiography, single-incision laparoscopic cholecystectomy

\section{Introduction}

Single-incision laparoscopic cholecystectomy (SILC), which was first introduced by Navarra et al. (1), is increasingly gaining popularity presumably because SILC has definite advantage in improving cosmesis after laparoscopic cholecystectomy (LC) $(2,3)$. In some institutions favoring SILC over conventional multiport laparoscopic cholecystectomy (CLC), incidence of SILC reached $50 \%$ among all the LC procedures $(4,5)$. In contrast, SILC has potential disadvantages compared to CLC in terms of longer operative time (6), increased cost for specialized laparoscopic instruments (7), a higher bile duct injury rate (8), and occurrence of umbilical hernia

\footnotetext{
*Address correspondence to:

Dr. Norihiro Kokudo, Hepato-Biliary-Pancreatic Surgery Division, Department of Surgery, Graduate School of Medicine, The University of Tokyo, 7-3-1 Hongo, Bunkyoku, Tokyo 113-8655, Japan.

E-mail: KOKUDO-2SU@h.u-tokyo.ac.jp
}

$(3,9)$. In order to safely adopt SILC in clinical practice, we need strict criteria for indication of SILC, which have not been indicated clearly even in the guideline of LC by the Society of American Gastrointestinal and Endoscopic Surgeons (10).

In the authors' institution, SILC has been performed after setting definite exclusion criteria comprising patients' background factors and gallstones/cholecystitis conditions. The present study evaluated intra- and postoperative outcomes of SILC compared to those of CLC with the aim to consider appropriate criteria for indicating SILC safely to patients who opt for singleincision surgery.

\section{Materials and Methods}

\subsection{Patients}

The subjects consisted of 146 consecutive patients who underwent LC for cholecystolithiasis, gallbladder 
polyps, or acute cholecystitis at The University of Tokyo between December 2009 and April 2012. Exclusion criteria for SILC were determined as follows: age $>75$ years, obesity $\left(\mathrm{BMI}>30 \mathrm{~kg} / \mathrm{m}^{2}\right)$, operative scar in the upper abdomen, concomitant cardiopulmonary diseases, acute cholecystitis, choledocholithiasis, abnormal bile duct anatomy detected by routine preoperative cholangiography on computed tomography, magnetic resonance images, or fluoroscopy. These criteria were set to secure the safety based on the studies which indicated higher postoperative morbidity rate following CLC for patients with higher age (11), the higher grade in American Society of Anesthesiologists score (11), and complicated and/or acute gallstone disease (11-13). SILC was indicated for patients who met the criteria and opted for SILC after being presented explanation on the potential benefits and disadvantages of this procedure. CLC and SILC were performed or guided by surgeons who have experienced general surgery and LC for more than 10 years. All operations were performed after obtaining informed consent from each patient, and patient anonymity was preserved.

\subsection{Surgical techniques}

For CLC, patients were placed in the supine position under general anesthesia as well as epidural anesthesia. The trocar for laparoscopy was placed via the umbilical region, and 2 to 3 trocars were generally placed on the epigastric region and on the right side of the umbilicus. For SILC, on the other hand, patients were placed in the supine position with legs spread apart under general anesthesia with local anesthesia around the umbilicus. SILS port (Covidien, Mansfield, MA, USA) and a 5.4 $\mathrm{mm}$-flexible laparoscope were used. A $2 \mathrm{~mm}$ visceral tractor (Mini Loop Retractor II; Covidien) was also used to lift the fundus of the gallbladder (14). The procedure was performed through Semi-cross method, holding an articulating instrument (Roticulator Endo Grasp; Covidien) with the left hand and a straight instrument with the right hand (15). The triangle of Calot was dissected to reach "critical view of safety" (16), while using fluorescence cholangiography (1720) with preoperative and intravenous injection of ICG at any time during the procedures (Figure 1 and supplementary video 1, http://biosciencetrends. com/docindex.php? year $=2015$ \&kanno $=6$ ). When the isolation of the cystic duct was not completed within one hour after the incision, the second trocar was then placed to facilitate laparoscopic procedures. During this study period, intraoperative drip infusion cholangiography using meglumine iotroxate (21) (Figure 2) as well as fluorescence cholangiography was also routinely performed for both LC and SILC at the authors' institution. The cystic duct was ligated and divided after confirming the anatomy of the common bile duct using drip infusion cholangiography and

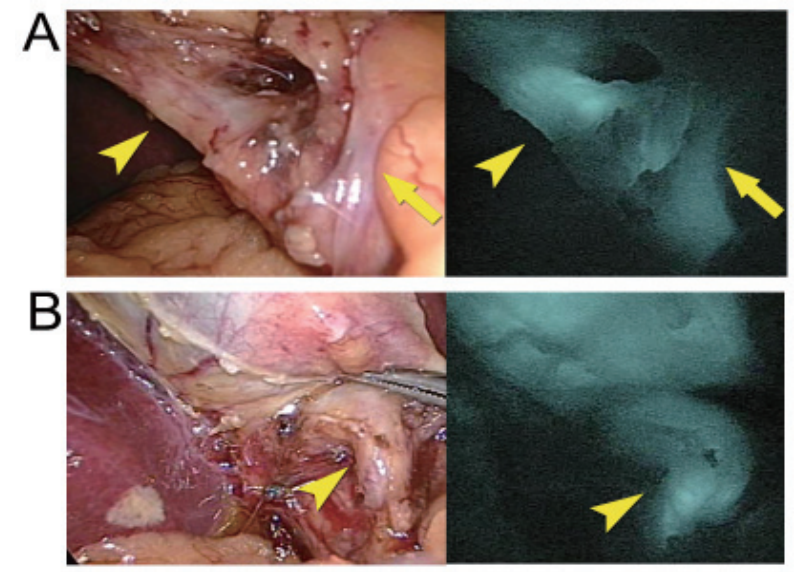

Figure 1. Fluorescence cholangiography during SILC. (A) Fluorescence cholangiography (right) after dissection of the triangle of Calot identifies the cystic duct (arrow head) and the common bile duct (arrow). (B) The cystic duct (arrow) is isolated and clearly visualized on fluorescence images. Please see supplementary video 1 .

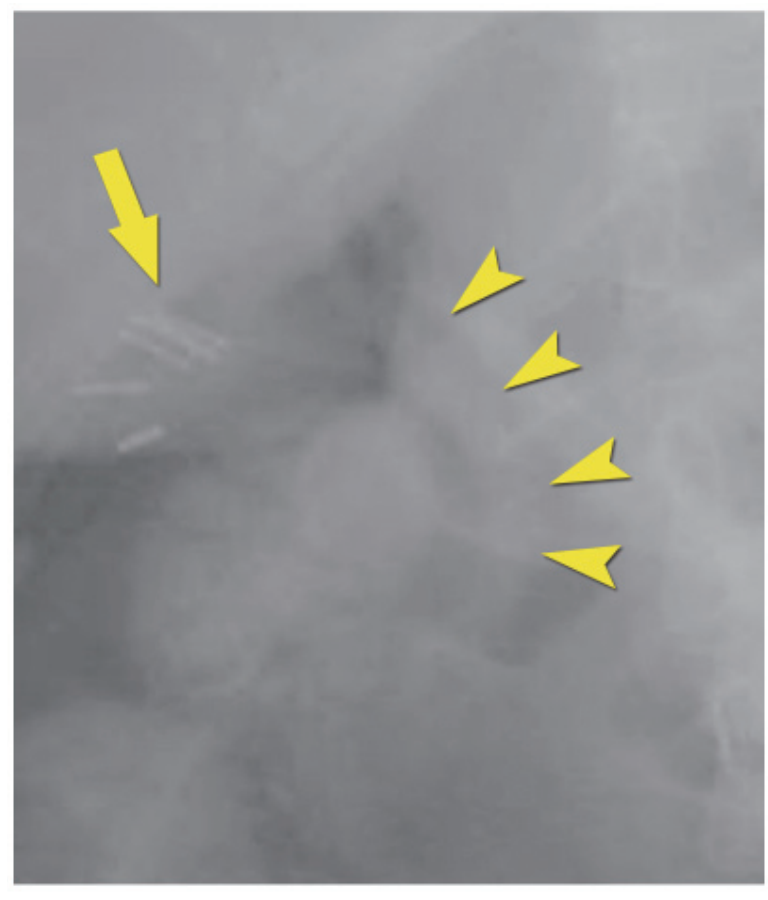

Figure 2. Drip infusion cholangiography. Drip infusion cholangiography was performed to confirm the relationship between the common bile duct (arrowheads) and clips made at the cystic duct (arrow).

fluorescence cholangiography, and consequently, the gallbladder was removed from the liver and retrieved. In principle, a drainage tube was not placed.

\subsection{Postoperative management}

The catheter for the epidural anesthesia in CLC was removed on postoperative day 2 . Non steroid antiinflammatory drug was administered intravenously or orally to relieve postoperative pain depending on patients' complaint. Patients were discharged from the hospital when levels of C-reacitive protein and hepato- 
biliary enzyme in blood were confirmed to be within normal limit on PODs 1 and/or 3 and when they had recovered from impaired activities of daily living. Follow-up was conducted for at least 6 months after surgery.

\subsection{Statistical analysis}

The subjects were divided into the following three groups: patients who met the exclusion criteria for SILC and underwent CLC (moderate/high-risk CLC group), those who were potential candidates of SILC but underwent CLC (Low-risk CLC group), and those who were potential candidates of SILC and actually underwent SILC (SILC group). Background characteristics and postoperative outcomes were compared between the Low-risk CLC group and the SILC group. Intraoperative factors were compared among the low-risk CLC group, the moderately/highrisk CLC group, and the SILC group.

Categorical variables are expressed in numerical figures (\%), and were compared between groups using Fisher's exact test or the chi-square test. Continuous variables were expressed as median values (with range), and were compared using the Wilcoxon's rank-sum test. In the analysis of intraoperative factors among the three groups, the Steel-Dwass test was used following Kruskal-Wallis test. $p$ values $<0.05$ were considered as denoting statistical significance. Statistical analysis was conducted using JMP software (version 9.0.2; SAS Institute Inc., Cary, NC).

\section{Results}

\subsection{Background characteristics}

The treatment flow in the study population is shown in Figure 3. Among the 146 patients, 34 (23\%) patients were excluded from the candidates of SILC (the moderate/high risk-CLC group) due to high age ( $n=$ $13)$, obesity $(n=11)$, acute cholecystitis $(n=3)$, surgical scar in the upper abdomen $(n=2)$, and abnormal bile duct anatomy on preoperative cholangiography $(n=$ 5). Of the remaining 112 candidates of SILC, 23 (21 $\%)$ patients opted for SILC and 89 patients (79\%) underwent CLC. Between the 23 patients in the SILC group and the 89 patients in the low risk-CLC group, the former group included younger patients and larger ratio of female patients (Table 1).

\subsection{Intraoperative outcomes}

Intraoperative factors are summarized in Table 2. Operation time in the low-risk CLC group was significantly shorter than that in the SILC group (126 [72-240] $\min v s .171$ [113-286] $\min , p<0.01)$ and also than that in the moderate/high-risk CLC group

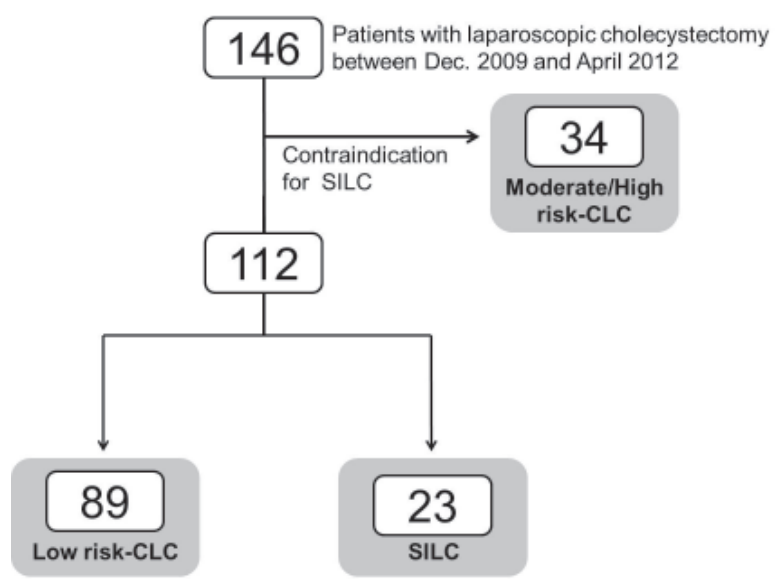

Figure 3. The treatment flow of study population. SILC, single-incision laparoscopic cholecystectomy; CLC, conventional multiport laparoscopic cholecystectomy; Low risk-CLC, CLC for patients who were the potential candidate of SILC but underwent CLC; Moderate/high risk-CLC, CLC for patients who met the exclusion criteria for SILC and underwent CLC.

Table 1. Background characteristics of SILC vs. low riskCLC

\begin{tabular}{lccc}
\hline Variables & $\begin{array}{c}\text { SILC } \\
(n=23)\end{array}$ & $\begin{array}{c}\text { Low risk-CLC } \\
(n=89)\end{array}$ & $p$ value \\
\hline $\begin{array}{l}\text { Age }(\mathrm{y})^{*} \\
\text { Gender }\end{array}$ & $45[19-65]$ & $59[22-75]$ & $<0.01$ \\
$\quad$ Female & $17(74 \%)$ & $39(44 \%)$ & $<0.01$ \\
$\quad$ Male & $6(26 \%)$ & $50(56 \%)$ & \\
BMI $\left(\mathrm{kg} / \mathrm{m}^{2}\right)^{*}$ & $21[16-30]$ & $23[16-30]$ & 0.116 \\
Indication & & & 0.308 \\
$\quad$ Stones & $23(100 \%)$ & $84(95 \%)$ & \\
$\quad$ Polypoid lesions & 0 & $3(3 \%)$ & \\
$\quad$ Both & 0 & $2(2 \%)$ & \\
\hline
\end{tabular}

BMI, body mass index. * Median [range].

Table 2. Intraoperative outcomes among SILC, low risk-CLC, and moderate/high risk-CLC

\begin{tabular}{lccc}
\hline Variables & SILC $(n=23)$ & Low risk-CLC $(n=89)$ & Moderate/High risk-CLC $(n=34)$ \\
\hline Operation time $(\mathrm{m})^{*}$ & $171[113-286]^{\mathrm{a}}$ & $126[72-240]^{\mathrm{a}, \mathrm{b}}$ & $150[100-343]^{\mathrm{b}}$ \\
Estimated blood loss $(\mathrm{mL})^{*}$ & $3[0-50]$ & $3[0-100] \mathrm{b}$ & $5[0-250]^{\mathrm{b}}$ \\
Additional trocar placement & $2(9 \%)$ & 0 & 0 \\
Conversions to laparotomy & 0 & $1(1 \%)^{\dagger}$ & $1(4 \%)^{\dagger}$ \\
Intraoperative complications & 0 & 0 & $1^{\ddagger}$
\end{tabular}

\footnotetext{
${ }^{*}$ Median [range]. $\dagger$ Intraoperatively diagnosed as gallbladder carcinoma. ${ }^{*}$ Anaphylaxis after intravenous injection of iodinated contrast material. ${ }^{a} p$
} $<0.05$ between the SILC group and the low-risk CLC group. ${ }^{\mathrm{b}} p<0.05$ between the low-risk CLC group and the moderate/high -risk CLC group. 
Table 3. Postoperative outcome of SILC vs. low risk-CLC

\begin{tabular}{lccc}
\hline Variables & SILC & Low risk-CLC & $p$ value \\
& $(n=23)$ & $(n=89)$ & \\
\hline $\begin{array}{l}\text { Postoperative complications } \\
\text { Use of postoperative painkiller }\end{array}$ & 0 & $2 \dagger$ & \\
None & $6(26 \%)$ & $11(12 \%)$ & 0.122 \\
$\leq 3$ days & $22(96 \%)$ & $46(52 \%)$ & $<0.01$ \\
Hospital stay (d)* & $2[2-4]$ & $4[2-12]$ & $<0.01$ \\
\hline
\end{tabular}

${ }^{*}$ Median [range]. ${ }^{\dagger}$ Pleural effusion $(n=1)$ and intra-abdominal abscess $(n=1)$.

(126 [72-240] min vs. 150 [100-343] min, $p<0.023)$. Estimated blood loss in the low-risk CLC was similar to that in the SILC group but smaller than that in the moderate/high-risk CLC group (low risk-CLC 3 [0100] $\mathrm{mL} v s$. moderate/high-risk CLC 5 [0-250] mL, $p=0.017)$. In the SILC group, additional trocars were needed in 2 patients $(9 \%$, one patient with BMI $29.9 \mathrm{~kg} / \mathrm{m}^{2}$ and severe adhesion around the liver and the gallbladder and the other patient with severe inflammation around the hepatoduodenal ligament). No intraoperative complication was observed in the SILC group. Conversion to laparotomy was needed for one patient (1\%) in the low-risk CLC group and one patient (4\%) in the moderate/high-risk CLC group due to the diagnosis of gallbladder carcinoma in the intraoperative pathological findings.

\subsection{Postoperative outcomes}

Postoperative complications (Clavien-Dindo classification [22] grade II or more) developed only in the Low risk-CLC group (pleural effusion and intra-abdominal abscess, Table 3). No bile leak was encountered in the SILC group or in the Low risk-CLC group. Postoperative hospital stay was significantly shorter in the SILC group than in the Low risk-CLC group (2 [2-4] days vs. 4 [2-12] days, $p<0.01)$. Patients in the SILC group needed postoperative for shorter periods compared to those in the Low risk-CLC group (Table 3).

\section{Discussion}

In the present study, LC has been performed in 146 consecutive patients using the definite exclusion criteria for SILC. According to our criteria, $77 \%$ of the patients undergoing LC were defined as potential candidates for SILC, and of those, 23 patients decided to undergo SILC. In the SILC group, additional trocars were needed in two patients (9\%) and no conversion to open surgery was required. Although operation time was significantly longer in the SILC group than in the low risk-CLC group, SILC led to better postoperative outcomes in terms of length of hospital stay and painkiller use most likely due to its less-invasiveness without increasing risk of operative complications compared to CLC. These results suggest that SILC can be applied safely to patients who prefer better cosmetic outcomes if appropriate indication criteria for SILC is established.

In the previous series, some authors have proposed exclusion criteria for SILC consisting of clinical factors such as BMI $>35$ or $40 \mathrm{~kg} / \mathrm{m}^{2}(4,22,23)$, cholecystitis $(4,22)$, abdominal scar $(4,22,23)$, choledocholithiasis $(4,23)$, pacemaker (22), and pregnancy (22) (Table 4). Since operation time of SILC tend to be longer than that in the CLC, it is reasonable to consider patients with a high risk of general anesthesia and clinical factors that can further prolong operation time as contraindications for SILC. Since operation time of SILC tend to be longer than that in the CLC, it is reasonable to consider patients with a high risk of general anesthesia and clinical factors that can further prolong operation time as contraindications for SILC. The long operation time in our series was most likely due to unskilled surgical skills for single incision technique in addition to the application of intraoperative ICG-fluorescence imaging. In the present series, on the other hand, we regarded patients as having moderate/high risk of LC and contraindication for SILC if they met any of the followings: higher age, obesity, operative scar in the upper abdomen, concomitant cardiopulmonary diseases, acute cholecystitis, choledocholithiasis, and abnormal bile duct anatomy. Indeed, operation time and estimated blood loss in the moderate/high-risk CLC group were unfavorable compared to those in the low-risk CLC group, suggesting that our criteria may serve to assure safety of SILC. In contrast, our indication criteria for SILC are stricter than those used in the previous series in terms of restrictions in age and BMI. In particular, routine use of preoperative cholangiographic images to evaluate bile duct anatomy may be a debatable issue, but we believe that preoperative cholangiography and/or intraoperative fluorescence cholangiography $(18,19)$ is essential for reducing risk of bile duct injury especially in patients undergoing SILC. As singleincision surgery techniques advance, indications of SILC can be expanded gradually in future.

Interestingly, only $20 \%$ of potential candidates of SILC decided to undergo SILC after informed consent, most likely because the majority of patients in the present series prioritized safety of surgery over the advantages SILC demonstrated thorough randomized controlled trials $(2,3)$, including better cosmetic outcomes, less postoperative pain, and shorter length of hospital stay compared to CLC. If further advancements in surgical techniques, such as robotic surgery (24) and intraoperative fluorescence cholangiography (1720,25 ), enhance safety of SILC, the role of SILC in the treatment of cholecystolithiasis/cholecystitis may gain more presence in future.

In conclusion, SILC can be safely applied to patients 


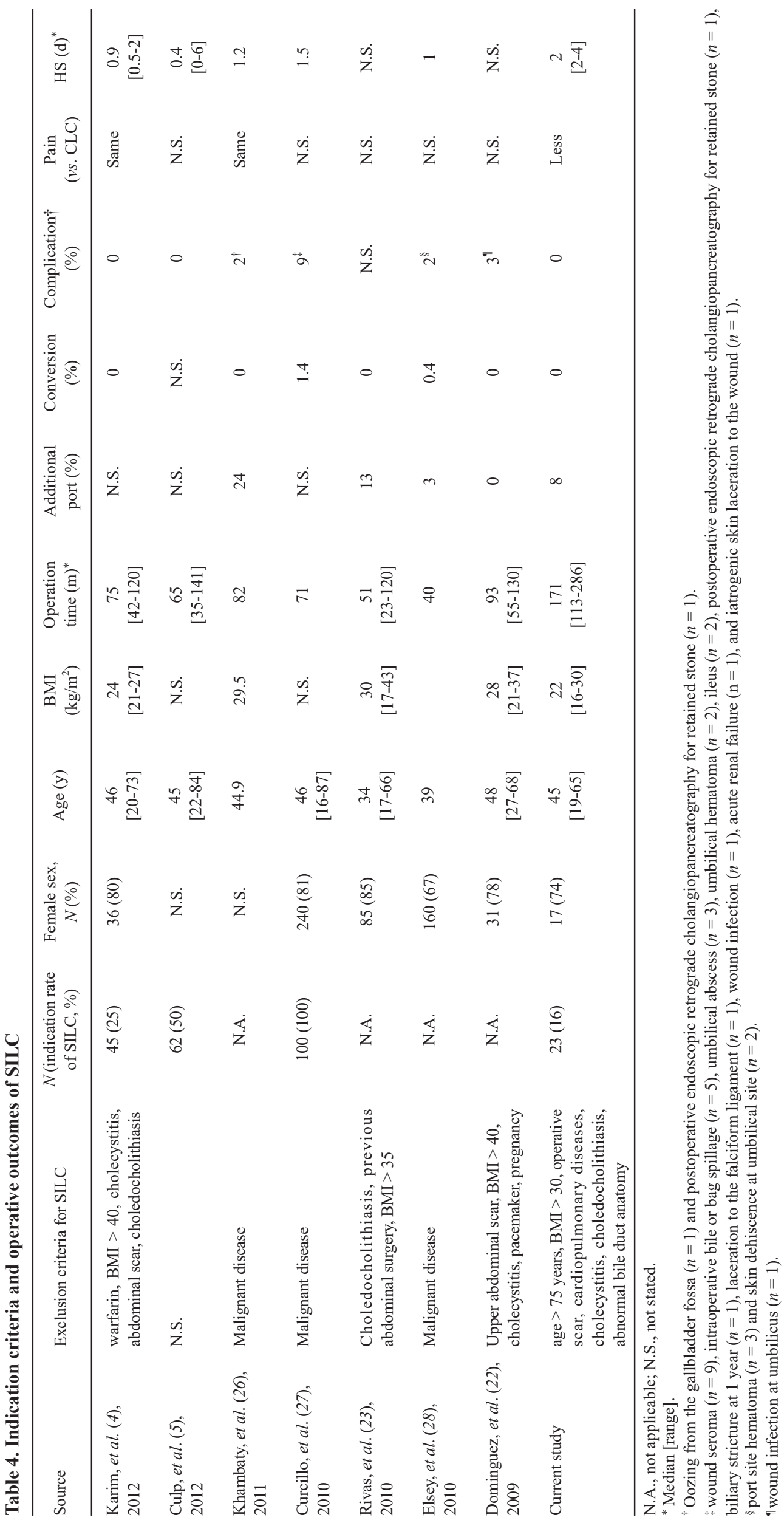


who desire better cosmetic outcomes if appropriate exclusion criteria are established through consideration of potential risks that SILC poses in prolonged operation time and bile duct injury.

\section{Acknowledgements}

This work was supported by grants from the Takeda Science Foundation, the Kanae Foundation for the Promotion of Medical Science, and the Ministry of Education, Culture, Sports, Science and Technology of Japan (No. 23689060 and No. 23249067).

\section{References}

1. Navarra G, Pozza E, Occhionorelli S, Carcoforo P, Donini I. One-wound laparoscopic cholecystectomy. Br J Surg. 1997; 84:695.

2. Bucher P, Pugin F, Buchs NC, Ostermann S, Morel P. Randomized clinical trial of laparoendoscopic single-site versus conventional laparoscopic cholecystectomy. Br J Surg. 2011; 98:1695-1702.

3. Marks JM, Phillips MS, Tacchino R, Roberts K, Onders R, DeNoto G, Gecelter G, Rubach E, Rivas H, Islam A, Soper N, Paraskeva P, Rosemurgy A, Ross S, Shah S. Single-incision laparoscopic cholecystectomy is associated with improved cosmesis scoring at the cost of significantly higher hernia rates: 1-year results of a prospective randomized, multicenter, single-blinded trial of traditional multiport laparoscopic cholecystectomy $v s$ single-incision laparoscopic cholecystectomy. J Am Coll Surg. 2013; 216:1037-1047; discussion 1047-1038.

4. Karim MA, Ahmed J, Mansour M, Ali A. Single incision $v s$. conventional multiport laparoscopic cholecystectomy: A comparison of two approaches. Int J Surg. 2012; 10:368-372.

5. Culp BL CV, Arnold DT. Single-incision laparoscopic cholecystectomy versus traditional four-port cholecystectomy. Proc (Bayl Univ Med Cent). 2012; 25:319-323.

6. Aprea G, Coppola Bottazzi E, Guida F, Masone S, Persico G. Laparoendoscopic single site (LESS) versus classic video-laparoscopic cholecystectomy: A randomized prospective study. J Surg Res. 2011; 166:e109-112.

7. Henriksen NA, Al-Tayar H, Rosenberg J, Jorgensen LN. Cost assessment of instruments for single-incision laparoscopic cholecystectomy. JSLS. 2012; 16:353-359.

8. Joseph M, Phillips MR, Farrell TM, Rupp CC. Single incision laparoscopic cholecystectomy is associated with a higher bile duct injury rate: A review and a word of caution. Ann Surg. 2012; 256:1-6.

9. Erdas E, Dazzi C, Secchi F, Aresu S, Pitzalis A, Barbarossa M, Garau A, Murgia A, Contu P, Licheri S, Pomata M, Farina G. Incidence and risk factors for trocar site hernia following laparoscopic cholecystectomy: A long-term follow-up study. Hernia. 2012; 16:431-437.

10. Guidelines for the Clinical Application of Laparoscopic Biliary Tract Surgery: Society of American Gastrointestinal and Endoscopic Surgeons. http:// wwwsagesorg.

11. Veen EJ, Bik M, Janssen-Heijnen ML, De Jongh M, Roukema AJ. Outcome measurement in laparoscopic cholecystectomy by using a prospective complication registry: Results of an audit. Int J Qual Health Care. 2008; 20:144-151.

12. Georgiades CP, Mavromatis TN, Kourlaba GC, Kapiris SA, Bairamides EG, Spyrou AM, Kokkinos CN, Spyratou CS, Ieronymou MI, Diamantopoulos GI. Is inflammation a significant predictor of bile duct injury during laparoscopic cholecystectomy? Surg Endosc. 2008; 22:1959-1964.

13. Kholdebarin R, Boetto J, Harnish JL, Urbach DR. Risk factors for bile duct injury during laparoscopic cholecystectomy: A case-control study. Surg Innov. 2008; 15:114-119.

14. Abe N, Takeuchi H, Ueki H, Yanagida O, Masaki T, Mori T, Sugiyama M, Atomi Y. Single-port endoscopic cholecystectomy: A bridge between laparoscopic and translumenal endoscopic surgery. J Hepatobiliary Pancreat Surg. 2009; 16:633-638.

15. Chiu CG, Nguyen NH, Bloom SW. Single-incision laparoscopic appendectomy using conventional instruments: An initial experience using a novel technique. Surg Endosc. 2011; 25:1153-1159.

16. Strasberg SM, Hertl M, Soper NJ. An analysis of the problem of biliary injury during laparoscopic cholecystectomy. J Am Coll Surg. 1995; 180:101-125.

17. Aoki T, Murakami M, Yasuda D, Shimizu Y, Kusano T, Matsuda K, Niiya T, Kato H, Murai N, Otsuka K, Kusano M, Kato T. Intraoperative fluorescent imaging using indocyanine green for liver mapping and cholangiography. J Hepatobiliary Pancreat Sci. 2010; 17:590-594

18. Ishizawa T, Bandai Y, Ijichi M, Kaneko J, Hasegawa K, Kokudo N. Fluorescent cholangiography illuminating the biliary tree during laparoscopic cholecystectomy. $\mathrm{Br}$ J Surg. 2010; 97:1369-1377.

19. Ishizawa $T$, Kaneko J, Inoue $Y$, Takemura N, Seyama Y, Aoki T, Beck Y, Sugawara Y, Hasegawa K, Harada N, Ijichi M, Kusaka K, Shibasaki M, Bandai Y, Kokudo N. Application of fluorescent cholangiography to singleincision laparoscopic cholecystectomy. Surg Endosc. 2011; 25:2631-2636.

20. Kawaguchi Y, Ishizawa T, Masuda K, Sato S, Kaneko J, Aoki T, Beck Y, Sugawara Y, Hasegawa K, Kokudo N. Hepatobiliary surgery guided by a novel fluorescent imaging technique for visualizing hepatic arteries, bile ducts, and liver cancers on color images. J Am Coll Surg. 2011; 212:e33-39.

21. Ochiai T, Yamazaki S, Ohta K, Takahashi M, Iwai T, Irie T, Noguchi N, Takamatsu S, Kawamura T, Teramoto $\mathrm{K}$, Arii S. Is drip infusion cholecystocholangiography (DIC) an acceptable modality at cholecystectomy for cholecystolithiasis, considering the frequency of bile duct maljunction and intraoperative bile duct injury? J Hepatobiliary Pancreat Surg. 2004; 11:135-139.

22. Dominguez G, Durand L, De Rosa J, Danguise E, Arozamena C, Ferraina PA. Retraction and triangulation with neodymium magnetic forceps for single-port laparoscopic cholecystectomy. Surg Endosc. 2009; 23:1660-1666.

23. Rivas H, Varela E, Scott D. Single-incision laparoscopic cholecystectomy: Initial evaluation of a large series of patients. Surg Endosc. 2010; 24:1403-1412.

24. Buchs NC, Pugin F, Azagury DE, Jung M, Volonte F, Hagen ME, Morel P. Real-time near-infrared fluorescent cholangiography could shorten operative time during 
robotic single-site cholecystectomy. Surg Endosc. 2013; 27:3897-3901

25. Ishizawa $\mathrm{T}$, Tamura $\mathrm{S}$, Masuda $\mathrm{K}$, Aoki T, Hasegawa $\mathrm{K}$, Imamura H, Beck Y, Kokudo N. Intraoperative fluorescent cholangiography using indocyanine green: A biliary road map for safe surgery. J Am Coll Surg. 2009; 208:e1-4.

26. Khambaty F, Brody F, Vaziri K, Edwards C. Laparoscopic versus single-incision cholecystectomy. World J Surg. 2011; 35:967-972.
27. Curcillo PG, 2nd, Wu AS, Podolsky ER, et al. Singleport-access (SPA) cholecystectomy: A multi-institutional report of the first 297 cases. Surg Endosc. 2010; 24:1854-1860.

28. Elsey JK, Feliciano DV. Initial experience with singleincision laparoscopic cholecystectomy. J Am Coll Surg. 2010; 210:620-624, 624-626.

(Received October 27, 2015; Revised December 21, 2015; Accepted December 27, 2015) 